\title{
Complications of venepuncture
}

\author{
Omiepirisa Yvonne Buowari
}

Medical Women Association of Nigeria, Rivers State Branch, Port Harcourt, Nigeria

Email: yvonnebuowari@yahoo.com

Received 20 November 2012; revised 27 December 2012; accepted 15 January 2013

\begin{abstract}
Venepuncture is the commonest procedure performed in health care settings. A review of literature and search on complications of venepuncture and blood collection. This procedure is without complications, which sometime can be fatal. Complications that can arise from venepuncture include haematoma formation, nerve damage, pain, haemaconcentration, extravasation, iatrogenic anaemia, arterial puncture, petechiae, allergies, fear and phobia, infection, syncope and fainting, excessive bleeding, edema and thrombus.
\end{abstract}

Keywords: Venupucture; Haematoma; Complications; Syncope

\section{INTRODUCTION}

Venepuncture is the most common invasive medical procedure performed by health care providers [1,2]. Early detection and good communication between the patient and health care provider is important whenever venepucture is performed to prevent serious complications. Venepuncture is the act of puncturing the vein for giving a drug or removal of blood [2,3]. Venepuncture has been practiced for centuries and is still one of the most common invasive procedures in health care [2]. However, practices vary considerably between countries and between institutions and individuals within the same country. These differences include variations in blood sampling technique, use of safety devices, disposal methods, disposal methods, reuse of devices and availability of hepatitis b vaccine [2].

Venepuncture should be performed with care. Universal precautions should be observed at all times to protect the patient and health care provider. Gloves should always be worn when obtaining blood specimens. Before beginning any procedure, identify the patient accurately [3]. The veins of a patient are the main source of specimens for testing the entry point of medications and the site for intravenous infusion and blood transfusions because there are only a limited number of easily accessible veins in a patient, it is important that everything be done to preserve their good condition and availa- bility [3].

Blood can be collected from a patient for laboratory investigations, blood donation or therapeutically to reduce the amount of blood as in patients with polycythemia Vera. Blood investigation and analysis is one of the commonest diagnostic tool used by doctors.

A variety of adverse complications may be encountered during blood collection process [4]. Laboratory investigations request by doctors is an important component in the diagnosis and treatment of patients. A superficial vein most commonly used for venepuncture. The best sites for venepuncture of superficial veins of the upper limbs are the median cubital vein. It lies over the cubital fossa and serves as an anastomosis between the cephalic and basilic veins [5]. The cephalic vein in both the forearm and arm can be followed proximally where it empties into the axillary vein, the basilic veins joins the brachial vein [5]. Inappropriate sites for venepuncture are arm on side of mastectomy, edematous areas, haematomas, arms in which blood is being transfused, scarred areas, arms with fistulas or vascular grafts and sites above an intravenous cannular [5]. Serious complications can occur because of venepuncture even when only a small volume of blood is withdrawn; therefore, medical personnel should be prepared to provide appropriate care 1. Areas that have been burnt or scarred should be avoided during phlebotomy $[4,6]$. Burned areas are very sensitive and susceptible to infection whereas veins under scarred areas are difficult to palpate $[4,6]$ and difficult to insert needle [4].

In a study by Kagel and Rayan, the most common sites for developing complications in order of frequency are forearms, hand, wrist and antecubital fossa [7]. Patients whose veins have been repeatedly punctured often become scarred and feel hard when palpated [4].

\section{HEMATOMA FORMATION}

\subsection{Hematoma Formation Is the Most Common Complication of Venupuncture}

This type of complication is caused by blood leaking into the tissues during or after venepuncture. A common sign of this complication occurring is swelling at or near the 
venepuncture site.

Haematoma complication can occur as a minor complication of venepuncture 1. The haematoma forms under the skin adjacent to the puncture site [6]. When it occurs release the tourniquet immediately, withdraws the needle, and apply firm pressure [6]. Haematoma occurs when the area around the puncture sites begins to swell indicating that blood is leaking into the tissues, which will result in a bruise $[2,4]$ due to partial insertion into the vein 4 . If this happens, immediately remove the needles apply pressure for two minutes and recheck to ensure bleeding has stopped [4]. To avoid haematoma formation from venepuncture, puncture only the uppermost wall of the vein just under the skin, remove the tourniquets before removing the needle, use the major superficial veins, make sure the needle fully penetrates the uppermost wall of the veins because partial puncture may allow blood to leak into the tissue just under the skin and apply pressure to the puncture site. Haematoma is formed when blood leaks into the tissue surrounding the insertion site after failure to penetrate the vein properly during insertion [8]. Haematoma is a collection of blood under the skin [9]. If a haematoma begins to form while blood is being withdrawn, the needle should be removed immediately and pressure maintained over the site [9]. Some of the causes of haematoma formation after venepuncture are small fragile veins, needle too large, excessive probing to find vein, removing the needle prior to releasing the tourniquest, needle going all the way through the vein, needle only partially entering the vein allowing leakage, applying pressure to gauze before the needle is removed [9].

\subsection{Infection}

This may be localized giving rise to thrombophlebitis or systemic leading to septicemia. The infection may be localized or systemic. However, peripheral intravenous cannula infections are more commonly associated with localized than systemic infection [10]. Inadequate cleansing or poor technique can lead to infection [9]. Infection can cause phlebitis and thrombus formation [8]. It is prevented by good aseptic technique and keeping any dressing over the venepuncture site clean $[8,11]$. Phlebitis is an acute inflammation of the intima of the veins [8]. It is caused by mechanical and chemical irritation or by microscopic particles that may contaminate infusion fluids'.

\subsection{Nerve Damage}

Hematoma formation following venepucture can be painful and can potentially cause nerve damage [2,9]. Among complications associated with phlebotomy, nerve injury is relatively rare, but is potentially serious and often results in malpractice lawsuits [12]. Patient may feel sharp electric tingling if a nerve is hit. Immediate discontinue that venepuncture and the patient may need physical therapy [4]. Inappropriate sites or excessive probing may lead to nerve damage [9]. Patient may complain of severe pain and the procedure should be stopped immediately [9].

\subsection{Haemoconcentration}

This can result from prolonged tourniquet application. It can be caused by prolonged tourniquet application, massageing, squeezing or probing a site, long-term intravenous fluid therapy, scleroses or occluded veins, dehydration and certain diseases [4]. Haemoconcentration may cause false increase in potassium ion, phosphorus, ammonia and total protein [4]. Intravenous line complications can result in morbidity and increased health care costs from prolonged hospitalization, extended use of intravenous antibiotics therapy and surgical intervention [7].

\subsection{Extravasations}

Extravasations occurs when a cannula pulls out of the vein or becomes partly occluded by venous construction causing backflow of the infusate through the puncture site into the surrounding tissues [8]. The patient may complain if tightness, burning and discomfort around the intravenous site and there may be swelling and blanching of the tissues [8]. In extravagation, the cannula enters the tissues rather than the vein [11]. In this condition, flushing will be difficult and swelling/pain may be noted the cannula should be removed immediately.

\subsection{Syncope and Fainting}

Patients may become dizzy and faint at the thought or sight of blood [6]. It is caused because of rapid fall in the blood pressure and it is an autonomic nervous system reaction (psychomatic trigger) usually based on fear [6]. Syncope and fainting can procure during venepuncture $[1,4]$.

\subsection{Petechiae}

Petechiae may occur following venepuncture. This may be due to coagulation problems or abnormalities $[4,6]$. The patient may bleed excessively after blood collection; make sure bleeding stops prior to leaving the patient.

\subsection{Excessive Bleeding}

Excessive bleeding after venepucture can occur. In-patient on anticoagulants, on drugs such as aspirin medication or drugs that decrease the number of platelets [4]. The patient should not be left alone until bleeding has stopped. 


\subsection{Edema}

This is abnormal accumulation of fluids in the intercellular spaces of the body and it can be localized or diffused [4]. Collection of blood should be avoided from these sites, which will contaminate specimen with tissue fluid [4].

\subsection{Fear and Phobia}

Fear and phobia may occur in some patients on seeing the needle. This may cause the patient to move puncturing the artery rather than the vein [11]. The needle should be withdrawn and pressure applied.

\subsection{Thrombosis}

Thrombus is a solid mass derived from blood clot constituents in the vessels that is a clot. Thrombus may partially or fully occlude a vein or artery making venepuncture difficult [4].

\subsection{Arterial Puncture}

The artery can be punctured instead of the vein. When this occurs, the needle should be removed immediately and pressure applied over the site.

\subsection{Pain}

While some discomfort is to be expected, the needle should be removed immediately if the patient complains of excessive or severe pain [9].

\subsection{Allergies}

The patient may be allergic to the cleaning agent or other solutions used to disinfect the site therefore ask for any allergy. Some patients have latex allergy.

\subsection{Iatrogenic Anemia}

Anemia may occur when large volumes of blood are collected for investigations and is common in infants.

\section{REFERENCES}

[1] Gelena, H.J. (1992) Complications occurring from diagnostic venipuncture. The Journal of Family Practice, 34, 582-584.

[2] World Health Organization (2010) WHO guidelines on drawing blood: Best practices in phlebotomy. http://www.who.int/en/

[3] West Tennessee Health Care Integrated Laboratory. Venepuncture. Physicians' laboratory handbook. www.gomcl.com

[4] Complications in blood collection. Professional and Linguistic Assessments Board 1223/1023, 66-71.

[5] The BD Vascutainer Blood Collection System. Helping all people live healthy lives. http://www.bd.com/vascutainer

[6] Edtexx Medical Cooperation (2007) Fundamentals of phlebotomy. 2nd Edition, Texas Tech University, Lubbock, 43-53. www.depts.ttu.edu

[7] Kagel, E.M. and Rayan, G.M. (2004) Intravenous catheter complication in the hand and forearm. Journal of Trauma-Injury Infection \& Critical Care, 56, 123-127. doi:10.1097/01.TA.0000058126.72962.74

[8] University Section of Anesthesia, Pain and Critical Care Medicine. Venepuncture and intravenous cannulation. Clinical Skills, 16. www.gla.ac.uk

[9] Phlebotomists Association of Ireland Ltd. (2010) Phlebotomy guidelines. 25-26. www.pairl.ie/doc/pal_guidelines_pdf

[10] Morris, W. and Tay, M.H. (2008) Strategies for preventing peripheral intravenous cannula infection. British Journal of Nursing, 7, S14-S21.

[11] Ohrishi, H., Watanabe, M. and Watanabe, T. (2012) Butterfly needles reduce the incidence of nerve injury during phlebotomy. Archives of Pathology and Laboratory Medicine, 136, 352. doi:10.5858/arpa.2011-0431-LE

[12] Cole, E. Cannulation and venepuncture. http://www.cefl.org.uk/ 